\title{
The quantitative analysis of electric meter measurement error with harmonic sources
}

\author{
Xiyang OU ${ }^{1, a}$, Kongjun ZHOU ${ }^{1, b}$,Jin ZHANG $^{1, c}$, Xiang DU ${ }^{2, d}$, Xiao-zhe \\ TANG $^{2, e}$, Xi OU $^{1, f}$ \\ ${ }^{1}$ Sate Grid Chongqing Electric Power Research Institute, Yubei, Chongqing, 401123, China \\ 2 Chongqing YuDian Electric technology Co. LTD, Yubei, Chongqing, 401122, China \\ aoxyang2002@163.com, bczkj@163.com, '443713574@qq.com,dduxiang@163.com, \\ 13808379587@126.com, fleihongxia830@sohu.com
}

Keywords: Induction meter; Electronic energy meter; Harmonic; Measuring error

\begin{abstract}
With the development of power electronic technology, it has become the main harmonic sources in the grid. This paper is based on the theory of induction meter and electronic energy meter. First the mathematical expressions of respective meter under harmonic are induced, and next the influence factor of the electric meter measuring error is analyzed. The theory of induction electric meter and electronic energy meter is respectively utilized for simulation analysis. Finally, combined with theoretical calculation and simulation analysis, the conclusion on the impact of harmonics on electric meter measurement is drawn.
\end{abstract}

\section{Introduction}

Electric energy is indispensable and important energy commodity in modern social production and life [1]. There are trade settlement problems as well as other commodity. Therefore, the accuracy and rationality of electric energy measurement directly affect the electricity generation, supply and utilization. In recent years, the power electronic technology is widely used in the production and life. The nonlinear load such as wave power load, large capacity rectification equipment and electrified railway, which is arc furnace, intermediate frequency furnace and so on used in modern industrial production generated a lot of harmonic voltage and current which make the error of electric energy measurement device and lead to electric energy metering data inaccurate, finally affects the interests of three parties such as generation, supply and utilization and the rationality of the transaction. Thus, it is very necessary to fully analyze the influence of harmonic on electric energy measurement.

This article is based on the theory of induction meter and electronic energy meter. The influence of the electric energy measurement of two kinds of meters with harmonic will be analysed and researched on next.

\section{Operating characteristics of electric meter under harmonic}

\section{Induction meter}

Induction meter is the instrument which is use of induced current force between fixed ac magnetic field and movable conductor [2][3].

The voltage $u$ loading on the coil is assumed:

$$
u=\sum_{h \geq 1} U_{h} \cos \left(h w t-\theta_{u h}\right)
$$

The work magnetic flux of the coil $\phi_{u}$ based on the magnetic circuit model of voltage coil can be defined as: 


$$
\phi_{u}=\sum_{h \geq 1} \frac{U_{h} W_{u}}{Z_{u h} \psi_{u h}} \cos \left(h w t-\theta_{u h}-\beta_{u h}-\alpha_{u h}\right)=\sum_{h \geq 1} \phi_{u h} \cos \left(h w t-\gamma_{u h}\right)
$$

Where $\theta_{u h}$ is the initial phase of every voltage harmonic, and $\left|Z_{u h}\right|^{\prime} \beta_{u h}{ }^{\prime} \psi_{u h}$ ' $\alpha_{u h}$ are respectively impedance value, impedance angle, magnetic resistance and flux loss angle of voltage coil under different frequency, and $W_{u}$ is the turns of voltage coil.

The current $i$ through the current coil is assumed:

$$
i=\sum_{h \geq 1} I_{h} \cos \left(h w t-\theta_{i h}\right)
$$

The work magnetic flux of the coil $\phi_{i}$ based on the magnetic circuit model of current coil can be defined as:

$$
\phi_{i}=\sum_{h \geq 1} \frac{I_{h} W_{i}}{\psi_{i h}} \cos \left(h w t-\theta_{i h}-\alpha_{i h}\right)=\sum_{h \geq 1} \phi_{i h} \cos \left(h w t-\gamma_{i h}\right)
$$

Where $\theta_{i h}$ is the initial phase of every current harmonic, and $W_{i}$ is the turns of current coil, and $\psi_{i h}$, $\alpha_{i h}$ are magnetic resistance and flux loss angle of current coil under different harmonic.

Based on the electromagnetic induction law, the induced eddy current $i_{u}, i_{i}$ on the rotary table between voltage coil and current coil respectively can be:

$$
\begin{aligned}
& i_{u}=\sum_{h \geq 1} \frac{h w \phi_{u h}}{Z_{d h}} \sin \left(h w t-\gamma_{u h}-\alpha_{d h}\right) \\
& i_{i}=\sum_{h \geq 1} \frac{h w \phi_{i h}}{Z_{d h}} \sin \left(h w t-\gamma_{i h}-\alpha_{d h}\right)
\end{aligned}
$$

The average torque $T_{D}$ of rotary table is

$$
\begin{aligned}
& T_{D}=\frac{1}{2 \pi} \int_{0}^{2 \pi}\left(\phi_{u} i_{i}-\phi_{i} i_{u}\right) d(w t)=\sum_{h \geq 1} \frac{h w \phi_{u h} \phi_{i h} \cos \alpha_{d h}}{Z_{d h}} \sin \left(\gamma_{u h}-\gamma_{i h}\right) \\
& =\sum_{h \geq 1} \frac{h w \phi_{u h} \phi_{i h} \cos \alpha_{d h}}{Z_{d h}} \sin \left(-\varphi_{h}+\alpha_{u h}+\beta_{u h}-\alpha_{i h}\right) \\
& =\frac{h w \phi_{u 1} \phi_{i 1} \cos \alpha_{d 1}}{Z_{d 1}} \cos \varphi_{1}+\sum_{h>1} \frac{h w \phi_{u h} \phi_{i h} \cos \alpha_{d h}}{Z_{d h}} \cos \left(\varphi_{h}+\delta_{h}\right)
\end{aligned}
$$

Where $\varphi_{h}=\theta_{i h}-\theta_{u h}$ is the harmonic power angle, and $Z_{d h}{ }^{\prime} \alpha_{d h}$ is the impedance value and impedance angle of rotary table, $\delta_{h}=\frac{\pi}{2}+\alpha_{i h}-\alpha_{u h}-\beta_{u h}$.

Brake torque is mainly produced by rotary table cutting brake magnet flux, cutting work voltage and current magnetic flux also produce smaller braking torque

$$
T_{B}=\frac{1}{2 \pi} \int_{0}^{2 \pi}\left(k_{1} \phi_{m} i_{m}+k_{2} \phi_{u} i_{u}+k_{3} \phi_{i} i\right) d(w t)=n\left[k_{m}+k_{u} \frac{\sum_{h \nu 1} \cos \alpha_{d h}}{Z_{d h}}\left(\phi_{h h}\right)^{2}+k_{i} \frac{\sum_{h v 1} \cos \alpha_{d h}}{Z_{d h}}\left(\phi_{h h^{2}}\right)^{2}\right]
$$

When it stable, the speed of rotary table is

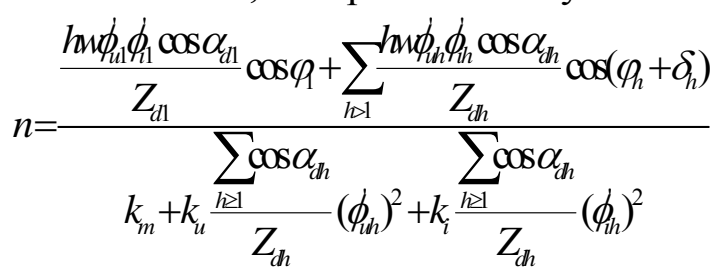

Where $k_{m}, k_{u}, k_{i}$ is respectively brake magnets, work voltage coil magnetic flux and current coil magnetic flux of the braking coefficient 
Compared to the brake coil, the braking effect of the voltage and current coil is minimal. So this paper is only considered by the braking effect of brake coil in the following analysis. The speed is

$$
\begin{aligned}
& n=\frac{\frac{h w \phi_{u 1} \phi_{i 1} \cos \alpha_{d 1}}{Z_{d 1}} \cos \varphi_{1}+\sum_{h>1} \frac{h w \phi_{u h} \phi_{i h} \cos \alpha_{d h}}{Z_{d h}} \cos \left(\varphi_{h}+\delta_{h}\right)}{k_{m}} \\
& =\frac{1}{k_{m}} \frac{w w_{u} w_{i} \cos \alpha_{d 1}}{Z_{u 1} Z_{d 1} \psi_{u 1} \psi_{i 1}}\left[U_{1} I_{1} \cos \varphi_{1}+\sum_{h>1} \frac{h Z_{u 1} Z_{d 1} \psi_{u 1} \psi_{i 1} \cos \alpha_{d h}}{Z_{u h} Z_{d h} \psi_{u h} \psi_{i h} \cos \alpha_{d 1}} U_{h} I_{h} \cos \left(\varphi_{h}+\delta_{h}\right)\right] \\
& =C\left[U_{1} I_{1} \cos \varphi_{1}+\sum_{h>1} k_{h} U_{h} I_{h} \cos \left(\varphi_{h}+\delta_{h}\right)\right]
\end{aligned}
$$

The error reasons of induction meter under the condition of harmonic are known from the analysis of the above :

Due to the magnetic impedance increased with the increase of frequency, so voltage coil and current coil magnetic flux is decreased with the increase of frequency;

The impedance between voltage coils and the rotary table is increased as the frequency;

There is harmonic power angle deviation.

Induction meter in the factory is debugged to make it only if the voltage and current is sinusoidal and the frequency of power frequency to show the best performance of electric energy metering accuracy. When the system voltage and current waveform is deviated from the sine wave or the frequency is changed, the induction meter measurement accuracy will decline. When there are other frequency signals in the voltage signal, the equivalent impedance and the impedance angle of the rotary table corresponding to the different frequency have different values, namely the speed under different frequencies is not corresponding to the same scale coefficient, and result in a measurement error.

In conclusion, with the electric energy metering in induction watt-hour meter, the following situations may occur:

(1) electric energy metering for linear load

a) When power supply is sine wave, watt-hour meter reflects the fundamental wave of the user consumption power.

b) When power supply is distorted, watt-hour meter reflects the fundamental wave of the user consumption power and part of the sum of harmonic power. That is to say, users will paying more, this time is against linear users.

(2) electric energy metering for nonlinear load

a) When power supply is sine wave, watt-hour meter reflects the numbers should be fundamental wave of the user consumption power minus the part of the user to the system harmonic power. The power charge will be less calculated for electricity power supply department at this time.

b) When power supply is distorted, watt-hour meter reflects the numbers should be fundamental wave of the user consumption power plus part of the power supply of harmonic power subtracting the user send a part of harmonic power to system. It is not conducive to the power supply department or the user. at this time.

\section{Electronic energy meter}

Electronic energy meter put voltage signal and current signal into the multiplier for instantaneous power with voltage sampling and current sampling, and for active power through the low-pass filter [4][5].

The voltage and current in a circuit is assumed as:

$$
\begin{aligned}
& u=\sum_{h \geq 1} U_{h} \cos \left(h w t-\theta_{u h}\right) \\
& i=\sum_{h \geq 1} I_{h} \cos \left(h w t-\theta_{i h}\right)
\end{aligned}
$$

The instantaneous power is:

$$
p=u i=\sum_{m \geq 1 n \geq 1} U_{m} I_{n} \cos \left(m \omega t-\theta_{u m}\right) \cos \left(n \omega t-\theta_{i n}\right)=\sum_{m \geq 1 n \geq 1} \sum_{m} U_{n}\left[\cos \left(m \omega t+n \omega t-\theta_{u m}-\theta_{i n}\right)++\cos \left(m \omega t-n \omega t+\theta_{u m}-\theta_{i n}\right)\right]
$$

After low pass filter, the dc component is received: 
$u=\sum_{h \geq 1} U_{h} I_{h} \cos \left(\theta_{u h}-\theta_{i h}\right)$

Where $\theta_{u h} 、 \theta_{i h}$ is the early phase of voltage and current with h subharmonic

In the actual calculation is to use the discrete to implement. In discrete calculation, the method is that the time interval is $\Delta t$.It is meaning that voltage, current signal is sampled within each $\Delta t$.The instantaneous power of sampling point $[6]$ is

$p=u(k) i(k)$

It is the sum of active power of every harmonic after low-pass filter.

Based on the above analysis, it shows that electronic energy meter measuring accuracy depends on A/D sampling, digital multiplier and low pass filter and other components. Relative to the induction watt-hour meter, electronic watt-hour meter in measuring the distortion signal shows obvious advantages. Due to the A/D sampling signal processing bandwidth is limited, so it is only suitable for several hundred frequency range of the electric energy measurement, meaning that the harmonic frequency is higher, especially the harmonic power measurement error has more under 20 times larger [7].

\section{Harmonic influence under experimental study}

This section will be on MATLAB graphic analysis of harmonics for impact of the induction meter and electronic watt-hour meter measurement accuracy based on the foregoing the error formula. So the analysis results are shown in Fig. 1 and Fig.2 .

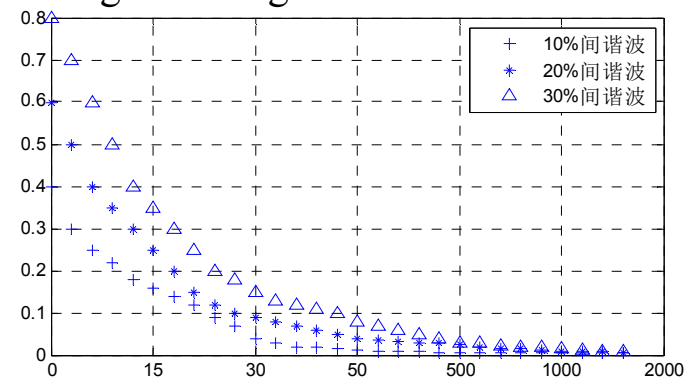

Fig. 1 Induction meter error on harmonic and frequency

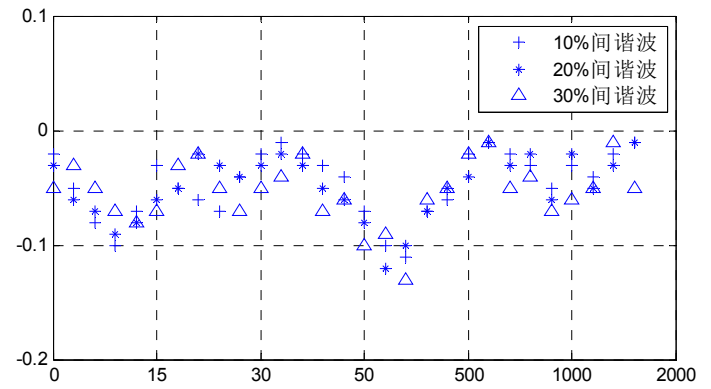

Fig. 2 Electronic energy meter error on harmonic and frequency

The Fig. 1 and Fig. 2 show the induction meter and electronic energy meter measuring error when the content of base wave respectively $10 \%, 20 \%$ and $20 \%$ of the harmonic in the frequency change from 0 to $2000 \mathrm{~Hz}$.

As shown in Fig.1 with the increase of frequency, induction electric meter measuring error decreases. When the harmonic frequency is big enough, the metering error basically ignored. This is mainly decided by the frequency characteristic of the induction meter. Because of its frequency characteristic is decayed with the increase of frequency, so that with the increase of frequency, the harmonic produced by the additional magnetic flux decreases, and the influence of the error on the electric metering is reduced gradually. Moreover, with the increase of the content of harmonic signals, the metering error is also gradually increased.

As shown in Fig. 2, for electronic energy meter, its frequency characteristics is basic stability with the increase of frequency within the scope of the bandwidth, so the figure shows the basic electric 
meter measuring error is zero. Even increasing the harmonic content of the signal also does not affect the overall error.

Comprehensive the above analysis, the results of this section analyses are same to ref. [8] based on the actual error of the meter change rule, which prove the correctness of the theoretical analysis in this paper.

\section{Conclusion}

Based on fully understanding induction meter and electronic energy meter principle, the mathematic expression of the electric meter is deduced under the condition of harmonic, and the influence of harmonic on the electric metering error is verified from two parts of theoretical analysis and experimental data. Simulation analysis shows that because of the induction meter is narrow width, the frequency response of harmonic generates larger impact on the performance of the measurement, and it is gradually decreased with the increase of the frequency. For the electronic meter, it has wide frequency band response because of the measurement principle of sampling and low pass filter. Whether the frequency is changed, it has smaller influence on energy meter measuring performance.

\section{References}

[1] Lin Xuemei. Harmonic problems in power system. Power Supply[J]. 2001, 18(3) 6-9.

[2] Baghzouz Y. et al, Harmonic analysis of induction watt-hour meter performance[J]. IEEE Trans. on Power Apparatus and Systems. 1985, 104(2): 399-406.

[3] Ding Yi, Zhao Wei, Hou Guoping. Induction meter measuring error analysis under the condition of harmonic[J]. Electric Measurement and Instrument. 2002, 39(442) 8-12.

[4] Wang Zhihua. Introduction to induction meter measurement[J]. Technology development. 2011, 30(12) 141-142.

[5] Luo Songtao. Quantitative analysis of the electronic watt-hour meter measurement error under the background of harmonic [D]. Zhengzhou University. 2012.

[6] Tang Hongyu. Design of Single-Phase Smart Meter Based on MSP430FE42X and ESP430 [J]. Low Voltage Apparatus, 2012(1) 41-45.

[7] Yang Tao. Design of High Accuracy Smart Meter System Based on ARM9 [J]. Low Voltage Apparatus, 2012(10) 56-58.

[8] Zhou Shaoyuan, Zheng Jiangzhong, Zhu Zhongwen. Study on the Effect of Interharmonics on the Electrical Energy Measurement of Watt-hour Metters [J]. The Fourth National Conference on electromagnetic measurement.2007(9) 331-335. 